\title{
Lack of correlation of P-glycoprotein expression with response to MIC chemotherapy in oesophageal cancer
}

\author{
S J Darnton, K Jenner, R S Steyn, D R Ferry, H R Matthews
}

\begin{abstract}
The multidrug resistance gene product $P$ glycoprotein (P-GP) was assessed immunohistochemically (by antibody JSB-1) in biopsy specimens from 27 oesophageal squamous carcinomas and 10 adenocarcinomas before treatment with mitomycin, ifosfamide and cisplatin (MIC). Tumours were assessed following treatment and correlation with response sought. Of the squamous carcinomas, $74 \%$ (20/27) responded to MIC but only one expressed P-GP before and after treatment. Of the adenocarcinomas, $30 \%$ (three of 10) responded. Seven of the 10 adenocarcinomas expressed P-GP before treatment but all 10 were $P$-GP positive after chemotherapy. The difference in prevalence and induction of P-GP between the histological types was highly significant and may correlate with the greater response to MIC seen in squamous carcinomas compared with adenocarcinomas. P-GP cannot be used as a predictive marker of response as tumours express it inconsistently with response to MIC. Resistance to MIC may be due to other mechanisms.

(f Clin Pathol 1995;48:1064-1066)
\end{abstract}

Keywords: Chemotherapy, multidrug resistance, oesophageal carcinoma, P-glycoprotein.

Cancer of the oesophagus has a poor prognosis (five year survival is less than $4 \%$ ). Survival of patients with squamous carcinoma is better than those with adenocarcinoma. Many patients have disseminated disease at presentation and most patients with resectable tumours eventually die of distant relapse. Chemotherapy potentially has a role in the control of systemic disease and could increase the chances of cure.

Results of phase II trials of neoadjuvant mitomycin, ifosfamide and cisplatin (MIC) show that squamous carcinomas are more responsive than adenocarcinomas (response rates $61 \%$ and $20 \%$, respectively).$^{12} \mathrm{~A}$ possible explanation for this difference could be the prevalence of the multidrug resistance gene (mdr-1) product, $\mathrm{P}$ glycoprotein (P-GP). One drug in the MIC regimen (mitomycin) is subject to transport out of cancer cells by P-GP. ${ }^{3}$ P-GP has been shown to play a part in primary and acquired resistance to chemotherapy in leukaemias, lymphomas, neuroblastomas, and childhood sarcomas ${ }^{4}$.

We investigated the prevalence of P-GP in prechemotherapy biopsy specimens and in posttreatment resected squamous carcinomas and adenocarcinomas of the oesophagus. Correlation of expression of P-GP with response to chemotherapy has been sought. Any such correlation might aid decisions about the suitability of neoadjuvant or adjuvant chemotherapy regimens.

\section{Methods}

Thirty seven patients (27 with squamous carcinomas and 10 with adenocarcinomas; table 1a), judged fit for curative surgery, received neoadjuvant MIC as described previously. ${ }^{1}$ Formalin fixed, paraffin wax embedded pretreatment biopsy specimens and post-treatment resected tumours were obtained in most cases. In six squamous carcinomas there was no tumour in the resected specimen (R0) and in one there was too little tumour remaining for assessment.

One $3 \mu \mathrm{m}$ section was stained with haematoxylin and eosin. Sections for immunohistochemistry were mounted on coated slides (Vectabond, Vector Laboratories, Peterborough, UK) and dried overnight at $37^{\circ} \mathrm{C}$. After rehydration, antigen retrieval involved placing slides in $0.01 \mathrm{M}$ citrate buffer $(\mathrm{pH} 6)$ and heating them in a microwave oven $(650 \mathrm{~W})$ for 15 minutes. Peroxidase activity was blocked with $0.6 \%$ hydrogen peroxide in methanol for 15 minutes. The Vectastain Elite ABC kit (Vector Laboratories) was used. The primary antibody was a mouse anti-human monoclonal, JSB-1 (diluted 1 in 20 in phosphate buffered saline (PBS), $\mathrm{pH} 7 \cdot 4$, and incubated for 30 minutes at room temperature), from Novocastra (Euro-Path Ltd, Bude, Cornwall, UK). The chromogen (brown staining) was 3, 3'-diaminobenzidine tetrahydrochloride (DAB Peroxidase Substrate kit, Vector Laboratories) with a light haematoxylin counterstain. The positive control was a pellet of cells, processed to wax, derived from a human small cell lung carcinoma cell line, H69LX4, known to express P-GP. ${ }^{5}$ Negative controls were sections from the cell line and test slides incubated in PBS alone.

Routinely stained sections were reported for histological type, depth of invasion and grade (the most poorly differentiated) according to the UICC. All sections were reported blind. Chemotherapy response was assessed on barium swallow. 
Table 1a Clinical details

\begin{tabular}{lll}
\hline $\begin{array}{l}\text { Histological } \\
\text { diagnosis }\end{array}$ & $\begin{array}{l}\text { Squamous } \\
\text { carcinoma }\end{array}$ & Adenocarcinoma \\
\hline Total number & 27 & 10 \\
Sex & $15 \mathrm{M}$ & $10 \mathrm{M}$ \\
Mean age (years)(range) & $62 \cdot 5$ & $61 \cdot 6$ \\
& $(26-77)$ & $(38-69)$ \\
Pulses of MIC & 18 & 10 \\
2 & 3 & - \\
3 & 6 & - \\
4 & & \\
Barium response after & 10 & 0 \\
2 pulses of MIC & 3 \\
$\quad$ complete responder & 10 & 7 \\
$\quad \begin{array}{l}\text { partial responder } \\
\text { non-responder }\end{array}$ & 7 & $30 \%$ \\
$\begin{array}{l}\text { Clinical response rate } \\
\text { (complete + partial }\end{array}$ & $74 \%$ & \\
responders) & &
\end{tabular}

Table 16 Histological results

\begin{tabular}{|c|c|c|}
\hline $\begin{array}{l}\text { Histological } \\
\text { diagnosis }\end{array}$ & $\begin{array}{l}\text { Squamous } \\
\text { carcinoma }\end{array}$ & Adenocarcinoma \\
\hline \multicolumn{3}{|c|}{ Pre-chemotherapy biopsy specimens } \\
\hline Total number & 27 & 10 \\
\hline \multicolumn{3}{|l|}{ Differentiation } \\
\hline G1 & 5 & 1 \\
\hline G2 & 12 & 6 \\
\hline G3 & 10 & 3 \\
\hline P-GP (+ve/-ve) & $1 / 26$ & $7 / 3$ \\
\hline \multicolumn{3}{|c|}{ Postchemotherapy resected cases } \\
\hline Total number & 27 & 10 \\
\hline \multicolumn{3}{|l|}{ Stage } \\
\hline 0 & 6 & 1 (TisNO) \\
\hline I & 1 & 0 \\
\hline IIA & 17 & 5 \\
\hline IIB & 1 & 0 \\
\hline III & 2 & 4 \\
\hline Total no. of tumours & 21 & 10 \\
\hline \multicolumn{3}{|l|}{ Differentiation } \\
\hline GI & 5 & 1 \\
\hline GI & 9 & 2 \\
\hline G3 & 7 & 7 \\
\hline \multicolumn{3}{|l|}{ Total no. for } \\
\hline immunohistochemistry & 20 & \\
\hline P-GP(+ve/-ve $)$ & $1 / 19$ & $10 / 0$ \\
\hline
\end{tabular}

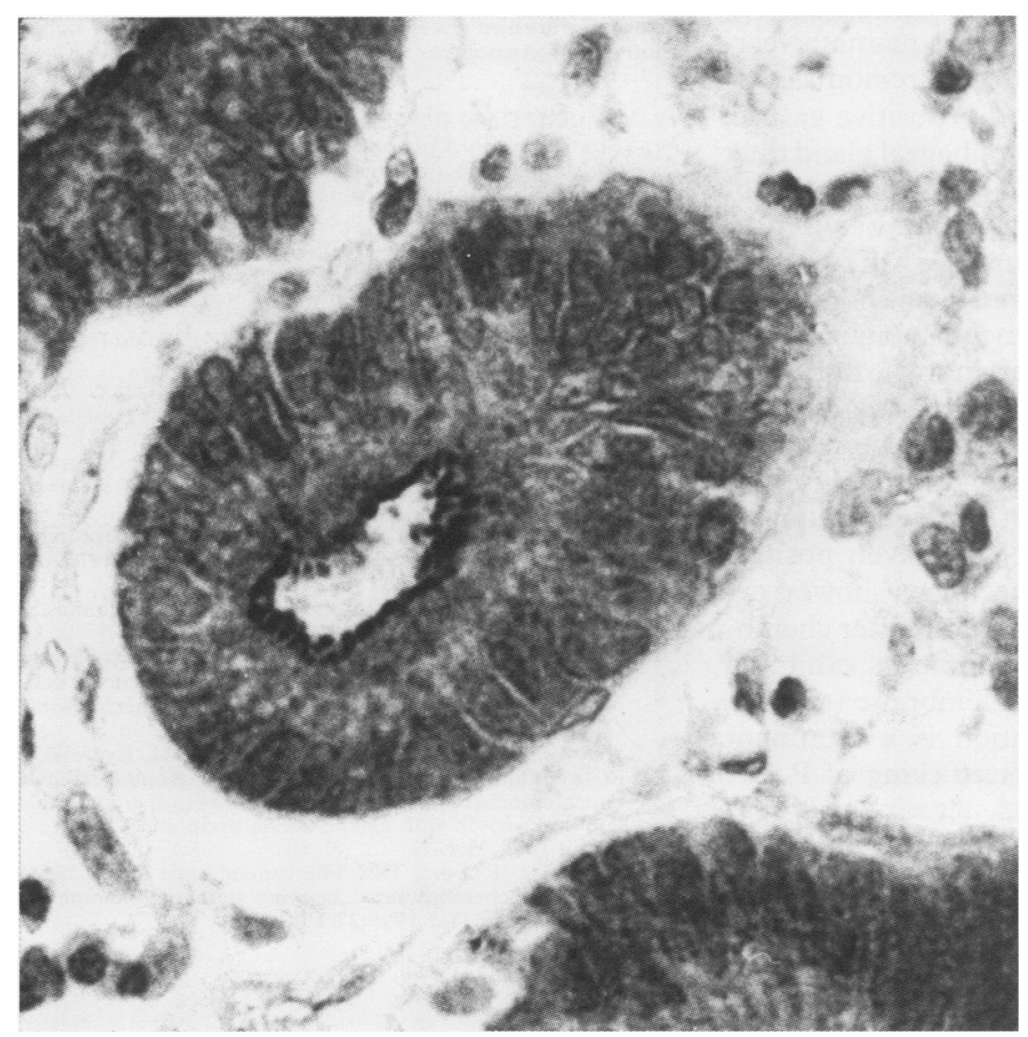

Treated, resected adenocarcinoma with cytoplasmic and apical membranous staining for $P-G P$.
Fisher's exact tests tested the hypothesis of no difference between the proportion of PGP positive biopsy specimens and resected tumours of the two histological types.

\section{Results}

Histological results are given in table $1 \mathrm{~b}$. Negative controls were unstained; the positive control cell line showed cytoplasmic and membranous expression of P-GP. Within the test sections macrophages, giant cells, endothelial cell cytoplasm, and some lymphocytes stained positively even when the malignant cells were negative.

Normal squamous epithelium was always negative. Dysplastic epithelium was negative where the tumour was negative but positive in the one positive case of squamous carcinoma. Glandular epithelium of the oesophageal glands and the stomach always showed cytoplasmic and membranous P-GP expression.

Pre- and post-chemotherapy squamous tumour samples were negative with the exception of one case in which both samples were positive. Before treatment, there was heterogeneous cytoplasmic staining (mainly of the Golgi region), but the treated tumour was homogeneously positive with cytoplasmic and cell membranous staining.

Before treatment, seven adenocarcinomas were P-GP positive and three negative. Six of the positive biopsy specimens showed cytoplasmic and membranous staining and one cytoplasmic only. All 10 treated adenocarcinomas, however, expressed P-GP with cytoplasmic and membranous staining (figure). There was no correlation between P-GP status and grade of biopsy or grade and staging of the resected tumours.

Statistical analysis demonstrated a highly significant difference $(p<0.0001)$ between the proportion of positive squamous biopsy specimens at $4 \%$ (95\% confidence interval (CI) $0 \cdot 1-19 \%)$ and the proportion of positive adenocarcinoma biopsy specimens at $70 \%$ (95\% CI 35-93\%), and a highly significant difference $(p<0.0001)$ between the proportion of positive resected squamous tumours at $5 \%$ $(95 \%$ CI $0 \cdot 1-25 \%)$ and the proportion of positive resected adenocarcinomas at $100 \%$ (95\% CI 69-2-100\%). Squamous carcinomas responded more frequently to MIC than adenocarcinomas (response rates of $74 \%$ and $30 \%$, respectively). There was no correlation between the P-GP status of the pre-treatment biopsy and the response to chemotherapy for either tumour type. Only one squamous carcinoma expressed P-GP before treatment (a partial responder). The other 26 patients with squamous tumours subsequently comprised 10 complete responders (six of which were R0), nine partial responders and seven non-responders. Of the seven positive prechemotherapy adenocarcinomas, two were partial and five were non-responders. Of the three negative pre-chemotherapy adenocarcinomas, one was a partial and two were non-responders. 


\section{Discussion}

Multidrug resistance phenotype, first described in 1970, is now known to be due to the presence of the trans-membrane glycoprotein P-GP, product of the gene mdr-1. Cells selected for resistance against one cytotoxic drug simultaneously acquire cross-resistance to a range of other natural cytotoxins. ${ }^{3-5}$ P-GP may efflux mitomycin but not ifosfamide and cisplatin from tumour cells. P-GP appears to be a highly conserved membrane transport protein and may be a common route for the export of environmentally occurring toxins. P-GP expression has been detected immunohistochemically in various epithelial tissues and it has been suggested that tumours arising from organs with high normal levels of expression might be intrinsically drug resistant. ${ }^{6}$ Tumours that initially respond to chemotherapy may be resistant on relapse and commonly show increase in the expression of P-GP. ${ }^{7}$

There have been few studies on P-GP in the normal oesophagus or oesophageal carcinomas. Saito $e t a l^{8}$ studied three cell lines from squamous carcinomas and found a pleiotropic mechanism of resistance without expression of $\mathrm{P}$ GP. Robey-Cafferty et al suggested that clinical response correlated with expression of P-GP in adenocarcinomas. ${ }^{9}$ They used an antibody less specific than JSB-1, C219, which also reacts with mdr-3 (not involved in drug resistance) and myosin. ${ }^{10}$ They did not always match pre-treatment biopsy specimens with treated tumours.

In the present study, excellent detection of P-GP was obtained in matched material. The staining pattern supports the concept that tumours originating from epithelia physiologically expressing P-GP, when transformed to a malignant state, retain expression. Squamous epithelium and most of the squamous carcinomas were negative. Adenocarcinomas, believed to originate from P-GP positive gastric, metaplastic Barrett's or oesophageal gland duct epithelia, all expressed P-GP after chemotherapy. No correlation was found between PGP expression and grade or staging of tumours.

Biopsy specimens from seven adenocarcinomas were homogeneously stained but that from one squamous carcinoma was heterogeneously positive. Sampling by a small biopsy alone could produce a false negative result in an heterogeneously expressing tumour.

The one positive biopsy specimen of squamous carcinoma and one of the seven positive adenocarcinoma biopsy specimens showed cytoplasmic staining only. However, after chemotherapy, the corresponding tumours exhibited both cytoplasmic and cell membrane staining. One consistent interpretation is a treatment induced alteration in cell trafficking of P-GP. The three adenocarcinomas, negative on biopsy but positive after chemotherapy, certainly represent increased P-GP expression (perhaps due to the natural history of treatment of the malig- nant cells or to a cell selection effect of the chemotherapy).

There was a highly significant difference between the proportion of positive biopsy specimens and tumours found in the two tumour types. The lower expression found in squamous carcinomas correlated with our experience of their better response to chemotherapy. The difference in survival between patients with untreated oesophageal squamous and adenocarcinomas suggests that the two tumours behave in a biologically different manner, the latter being inherently more aggressive. Origin from P-GP expressing normal tissues compounds this natural aggression in making adenocarcinomas more difficult to treat successfully with chemotherapy.

No relation has been found between the PGP status of the original biopsy specimen and the response of individual tumours. Non-responding tumours do not always express P-GP and responding tumours are sometimes P-GP positive. This suggests that resistance to $M I C$ is likely to involve other biochemical mechanisms associated with alkylating agents such as cellular concentrations of glutathione. ${ }^{10}$

The identification of potential individual responders to chemotherapy would be advantageous for clinical treatment. Patients with potentially resistant tumours could avoid unnecessary toxic treatment, proceeding directly to surgery. The lack of a correlation between P-GP expression in biopsy specimens and the response of tumours means that P-GP expression cannot be used as a predictive marker of response to MIC.

SJD, KJ and RSS are supported by the Oesophageal Cancer Research Appeal (OCRA), Birmingham. Statistical analysis was carried out by Ms L Billingham, The University of Birmingham Carried out by Ms L Billingham, The University of Birmingham CRC Institute for Cancer Studies, to whom we express our
thanks. We are also grateful to Mrs K H Whitehouse for skilled thanks. We are also grate
photographic assistance.

1 Allen SM, Duffy JP, Walker SJ, Darnton SJ, Cullen MH, Matthews HR. A phase II study of mitomycin, ifosfamide and cisplatin in operable and inoperable squamous cell carcinoma of the oesophagus. Clin Oncol 1994;6:91-5.

2 Allen SM, Duffy JP, Darnton SJ, Cullen MH, Matthews HR. A phase II study of mitomycin, ifosfamide and cisplatin in adenocarcinoma of the oesophagus. Cancer Chemother Pharmacol 1996 (in press)

3 Yusa K, Sato W, Yamazaki A, Tsukahara S, Tsuruo T. Cross-resistance of human multidrug-resistant cells to mitomycin C. Anticancer Res 1991;11:1301-4.

4 Ferry DR, Kerr DJ. Multidrug resistance in cancer. $B M F$ 1994;308:148-9.

5 Twentyman PR, Fox NE, Wright KA, Bleehen NM. Derivation and preliminary characterisation of adriamycin resistant lines of human lung cancer cells. $\mathrm{Br} \mathcal{F}$ Cancer 1986;53:529-37.

6 Endicott JA, Ling V. The biochemistry of P-glycoproteinmediated multidrug resistance. Ann Rev Biochem 1989; 58:137-71.

7 Yuen AR, Sikia BI. Multidrug resistance in lymphomas. $f$ Clin Oncol 1994;12:2453-9.

8 Saito T, Hikita M, Kohno K, Sato S, Takano H, Kobayashi $M$. Different sensitivities of human esophageal cancer cells to multiple anti-cancer agents and related mechanisms. Cancer 1992;70:2402-9.

9 Robey-Cafferty SS, Rutledge ML, Bruner JM. Expression of a multidrug resistance gene in esophageal adenocarcinoma. Correlation with response to chemotherapy and comparison with

10 Woodhouse JR, Ferry DR. The genetic basis of resistance to cancer chemotherapy: opening potential therapeutic to cancer chemotherapy: opening
avenues. Ann Med 1995;27:157-67. 